\title{
Damage by Induced Electric Field versus Radiolysis
}

\author{
Nan Jiang ${ }^{1}$ \\ ${ }^{1 .}$ Department of Physics, Arizona State University, Tempe AZ, USA.
}

Radiolysis has been the most referred mechanism to interpret damage phenomena in electron microscopy, especially in semiconductor and insulator materials. This type of damage is initiated from the electronic excitation and ionization by beam electrons. In radiation chemistry, the original concept of radiolytic process results in the cleavage of chemical bond(s) due to exposure to ionizing radiation, and forms radicals. These radicals are highly reactive. The main difference between radiolysis and ionization is that in the former the radical has at least one unpaired electron and this molecule or atom does not carry a charge, while in the latter the ion is either positively or negatively charged due to the loss or gain of an electron [1]. Obviously the radiolytic process in radiation chemistry is not the same as what we meant in TEM, in which beam damage is not caused by chemical reaction (with nearest rigid atoms) but by displacement of atom. In rigid solids, the mechanism should be able to provide explicitly how the atom acquires momentum to displace, rather than how the chemical bond breaks. Unfortunately, only a few have addressed this in details among a vast number of publications.

The first application of radiolysis in beam damage was to interpret defect formation in alkali and alkalineearth halides by ionization radiation, in which the radiolytic process was initiated by the creation of an exciton, and atomic displacement was driven by thermal vibration [2]. Another well-documented application is to interpret amorphization of $\mathrm{SiO}_{2}$ by an electron beam in TEM [3]. It was triggered by the point defects, which were radiolytically produced Frenkel type, consisting of $\mathrm{O}-\mathrm{O}$ and $\mathrm{O}$ vacancies [3]. The displacement of $\mathrm{O}$ atom was driven by Coulomb repulsive forces. This second is more significant, since the amorphization can be seen in situ in TEM. The formation of $\mathrm{O}-\mathrm{O}$ defects during ionization irradiation has been confirmed experimentally electron-spin-resonance measurements. However, the estimated concentration of $\mathrm{O}$ defects in the end products is equivalent to one damaged [ $\left.\mathrm{SiO}_{4}\right]$ tetrahedron among $10^{4}$ [4]. This estimate does not contradict EELS studies, in which $\mathrm{O}-\mathrm{O}$ defects has not been detected in any previous experiments [5], perhaps due to $\sim 0.01 \%$ concentration is too low to be detected using current instruments. $\mathrm{SiO}_{2}$ has the same $\left[\mathrm{SiO}_{4}\right]$ tetrahedral structure in both crystalline and amorphous forms, and it only loses the long-range order by beam damage. Considering that radiolytic process creates point defect locally as suggested in [3], the produce rate of $\mathrm{O}-\mathrm{O}$ defects should not be very different between crystalline and amorphous structures. Therefore it is reasonable to consider this $0.01 \%$ as the upper limit of radiolytically produced defects in $\mathrm{SiO}_{2}$. It is difficult to imagine that one defective tetrahedron can cause cascade collapse of $10^{4}$ ordered tetrahedra. Although in broad beam illumination (lower dose rate) amorphization of $\mathrm{SiO}_{2}$ occurred preferentially at surfaces and around existing defects, it was uniformly formed in the specimen when the focused beam (higher dose rate) was used [6].

Originated from the charging due to electronic excitation and ionization, the mechanism of damage by induced electric field (DIEF) has been introduced recently [5]. The main differences from the radiolysis are (1) the driving forces of atomic displacement are local electric fields; (2) the displaced atoms are not necessarily associated with the radiolytically formed defects; (3) the atomic displacements are not random but the same type has the similar movement; (4) damage is electron dose-rate dependent. The charging distribution is not uniform. Depending on illumination, the damage is therefore heterogeneous, preferentially located near surfaces, specimen edge [7], and as well as around existing defects. Two factors 
may facilitate the charge accumulation at defects. One is that they may trap holes produced by electron beam, and the other is that the line or plane defects may provide conducting paths for excited electrons to flow towards surfaces, driven by the positive potential built up at the surfaces. These mechanisms can well interpret amorphization of $\mathrm{SiO}_{2}$. Besides phase separation and phase transformation, the precipitation of metal nanoparticles is also an energetic favorable process under electron beam [8]. The Gibbs free energy barrier for nucleation of metal particles can be largely reduced under electron beam, and the directional drifting of atoms driven by the electric forces accelerates the kinetic process of metal particle precipitation as well.

The differences between radiolysis and DIEF have also been observed in liquid specimen. Unlike in solids, radicals do exist in liquid under electron beam irradiation. These highly reactive radicals can easily cause reactions and form new species inside liquid. Since the liquid TEM specimen usually contain two windows layers, such as $\mathrm{Si}_{3} \mathrm{~N}_{4}$ membrane, the induced electric fields are localized near the window region. Therefore, bubbles formed by DIEF mechanism mainly concentrated near the windows. The cryo-EM specimen consists of frozen water solution, and embedded supporting films such as lacy amorphous C. The damage can be more easily observed in the region containing $\mathrm{C}$ film than the region without $\mathrm{C}$. This phenomenon can be also interpreted by the DIEF mechanisms.

\section{References:}

[1] V Arena in "Ionizing Radiation and Life: An Introduction to Radiation Biology and Biological Radiotracer Methods", (St. Louis, Mosby).

[2] MN Kabler and RT Williams, Phys. Rev. B 18 (1978), p. 1948.

[3] LW Hobbs and MR Pascucci, Journal de Physique 41 (Colloque C6) (1980), p. 237.

[4] L Douillard and JP Duraud, Nucl. Instrum. Meth. Phys. Res. B 107 (1996), p. 212.

[5] N Jiang, Report on Progress in Physics 79 (2016), p. 016501.

[6] WL Gong et al., J. Appl. Phys. 81 (1997), p. 2570.

[7] N Jiang, Micron 83 (2016), p. 79.

[8] N Jiang, Ultramicroscopy 196 (2019), p. 18. 